\title{
FINITE ELEMENT MODELS FOR COMPUTER SIMULATION OF INTRASTROMAL PHOTOREFRACTIVE KERATECTOMY
}

\author{
AHMED A. HAMEED SAYED \\ Mechanical and Aerospace Department \\ West Virginia University, WV, USA \\ asayed@mix.wvu.edu \\ NAHED H. SOLOUMA \\ National Institute of Laser Enhanced Sciences \\ Cairo University, Giza, Egypt \\ AMAL A. EL-BERRY \\ National Research Center \\ Giza, Egypt \\ and \\ Information Systems Department \\ Faculty of Engineering and Computer Science \\ El-Kharj University, El-Kharj, KSA \\ YASSER M. KADAH \\ Biomedical Engineering Department \\ Cairo University, Giza, Egypt \\ http://www.k-space.org \\ ymk@k-space.org
}

Received 14 October 2010

Revised 29 November 2010

Accepted 17 January 2011

The main idea to correct sight disorders using lasers is to modify corneal curvature by applying laser to specific layers of the cornea. Intrastromal photorefractive keratectomy (ISPRK) is a laser technique used to correct sight disorders by evaporating corneal tissue from the stroma. Evaporating such tissue produces small cavities that may coincide to form a larger cavity. The composed big cavity is assumed to collapse to deform the overall curvature of the cornea. In this work, we provide finite element models to simulate the ISRPK procedure using a three-dimensional (3D) model of the cornea with typical parameters. The model outcome was compared with an earlier $2 \mathrm{D}$ model used for the same purpose, so as to determine its accuracy. In addition, a 3D finite element simulation of the procedure was made for a virtual astigmatic case to visualize the corneal curvature change. The results of this work show that this finite element models provide an accurate simulation of the corneal deformation expected after performing the procedure.

Keywords: ISPRK; photorefractive laser surgery; biomechanics; picosecond laser; femtosecond laser; finite element modeling. 


\section{Introduction}

Laser has been used in the treatment of eye diseases since 1962. The first use was for photocoagulation of retinal diseases. Latter, it was used to successfully treat glaucoma, which is a disease of increased intraocular pressure (IOP). Then, with the advent of Q-switching and mode locking, new horizons for using laser as a photodisruptor to incise tissue were opened. This paved the way for its use in removing secondary cataract without opening the eye. Laser is now largely used to correct refractive errors using photoablation to reform the shape of the cornea and helps people get rid of glasses.

Correction of refractive errors is done by reshaping the cornea because the cornea is the transparent portion of the eye, which allows light to enter and performs twothirds of the focusing tasks. The cornea covers both the iris, the colored portion of the external eye, and the pupil, which is the reactive "light meter" in front of the lens. Unlike the lens, the cornea's refraction power is fixed and it provides $65-75 \%$ of eye's focusing power since the anterior surface of the cornea is exposed to air with an index of refraction close to unity. Moreover, the cornea acts as a barrier protection and filtrates unwanted UV light ${ }^{1}$.

Corneal dimensions vary between $11.6 \mathrm{~mm}$ in the vertical direction and $11.5 \mathrm{~mm}$ in the horizontal direction. Its thicknesses are $0.65 \mathrm{~mm}$ at the limbus and $0.5 \mathrm{~mm}$ at the apex. ${ }^{2}$ The transparency of the corneal tissue in the spectral region from $400 \mathrm{~nm}$ to $1,200 \mathrm{~nm}$ can be attributed to its extremely regular microscopic structure. The optical zone of the human cornea has typical diameters ranging from $2 \mathrm{~mm}$ to $4 \mathrm{~mm}$ and is controlled by the iris.

There is no direct blood supply to the cornea, and that is to maintain its transparency. All nutrition is supplied indirectly through tears, ambient oxygen, and the aqueous humor of the anterior chamber. ${ }^{2}$

The cornea consists of five distinct layers: epithelium layer, Bowman's membrane, stroma, endothelium layer, and Descemet's membrane. The stroma, the supportive structure of the cornea, is composed of collagen fibrils and represents about $90 \%$ of the corneal thickness while the other four layers are for protection and maintenance.

Photorefractive surgical procedures are those used to reduce refractive errors such as myopia (nearsightedness), hyperopia (farsightedness), and astigmatism (distorted vision), by reshaping the flawed corneal surface. They represent exciting and extraordinary advances in the field of ophthalmology. All of these procedures are designed to minimize dependence on eyeglasses and contact lenses. Sight correction is done by influencing different types of tissues in the cornea, including portions of the epithelium, Bowman's membrane, and the stroma using laser ablation. Since the epithelium and the Bowman's membrane are the outer layers of the cornea, they prevent infection and smooth the refractive surface; it is preferable to leave them intact. Stroma is the supportive structure and the major part of the cornea responsible for its refractive properties because its thickness is about $90 \%$ of the 
total corneal thickness. Therefore, to get the best results of sight correction, tissue should be removed from the stroma to reform the cornea.

There are three main types of photorefractive surgery, namely, the photorefractive keratectomy (PRK), the laser-assisted in-situ keratomileusis (LASIK), and the intrastromal photorefractive keratectomy (ISPRK). In the PRK, the surface of the cornea is reshaped using Excimer laser, which means that the outer layers, the epithelium and the Bowmen's membrane, are influenced. Although it had reasonable success, the risk of scarring, unpredictable healing of the cornea, and possibility of infection still exist. LASIK is a procedure in which a thin surface flap of the cornea is created to expose the underlying tissues. It is within the deeper layers of the cornea that Excimer laser is applied to ablate a portion of the stroma and reshape the cornea. Once the laser ablation is completed, the surgeon gently replaces the corneal surface flap to restore surface integrity of the eye. LASIK has higher success rate than PRK with the primary potential risks include postoperative glare, halos, or starburst around lights at night and infection in the cornea with loss of best corrected visual acuity. ${ }^{3}$

Currently, a new approach of intrastromal tissue ablation is being under investigation. In this method, a femtosecond laser is used to ablate the required portion of the stroma without surgical intervention. This technique is called ISPRK and the first clinical results were obtained in 2003. Other names for this type of procedures also exist, e.g. femtosecond lenticule extraction (FLEx). ${ }^{4}$ The clinical trials showed that after surgery, the treated corneas were highly transparent and refractive results were stable. ${ }^{5}$ ISPRK is an interesting and challenging technique for refractive corneal surgery. The removal of tissue in the corneal stroma is performed by using a pulsed laser beam, which is sequentially focused to individual spots at a plurality of points in the stroma. A single layer of tissue is removed by steering about $10 \mu \mathrm{m}$ focal spot in a spiral pattern. The spots layers are arranged in successive spiral patterns to photo ablate and remove a plurality of layers of stromal tissue to form a cavity, with the diameters of the layers being properly sized to result in the desired diopter correction. ${ }^{6}$

The cavity has either a continuous disk-shaped for correcting myopic cases or a ring-shaped cavity that is generated to correct hyperopic cases. When the gaseous vapor inside the created cavity is diffused into the surrounding medium, the cavity collapses. It is expected that the removal of stromal tissue then induces a stable change in curvature of the anterior corneal surface. The main advantage of this technique is that the outer original layers of the cornea, mainly epithelium and Bowman's membrane, are not injured, which is not the case with Excimer laser surgeries. Thus, the stability of the cornea is less affected, and corneal haze is less likely to occur. ${ }^{2}$

In practice, however, it has been difficult to precisely and uniformly create intrastromal cavities using picosecond intrastromal ablation. Histological data performed on some eyes revealed a compartmentalized pattern of ablated tissue. 
Therefore, early clinical trials of the ISPRK surgery indicated some lack of control over the refractive results. ${ }^{7}$

A modified technique was suggested in which the laser is used to separate an intrastromal lenticule from the surrounding stroma, that only two intrastromal layers are removed: posterior and anterior surfaces of the lenticule. A flap is created in the anterior cornea, and the lenticule is removed. This technique is very similar to LASIK and has the same disadvantages. ${ }^{8,9}$

Experimental studies are currently being conducted to enhance and stabilize the intrastromal ablation procedures. In addition, as any laser surgery, the procedure needs to be quantized to obtain the optimum cavity dimensions and laser parameters that could be used to get the best correction results. Therefore, extensive theoretical models have been proposed to simulate this kind of surgery, especially using the algorithm of finite element modeling. The method of finite element modeling is a very powerful tool of modern engineering science. Reshaping of the cornea by mechanical alterations is a typical problem that can be studied by finite element modeling and certain predictions can be made concerning any changes in refraction. $^{2}$

Hennighausen and Bille ${ }^{10}$ created three finite element models to predict corneal curvature change after an ISPRK surgery. The results of the three models were compared. Their models differ essentially in the underlying assumptions about the mechanical behavior of the cornea. Another model developed by Bryant et al. ${ }^{8}$ in which they compared their finite element model results with a geometric model regarding clinical data. Both of the models have an error of about $20 \%$ compared with actual clinical data. Deenadayalu et al. ${ }^{11}$ used a similar finite element model to calculate refractive power change induced by LASIK surgery. They studied the effect of changing the flap dimensions, intraocular pressure, and modulus of elasticity on model results which concentrated on hyperopic cases.

In this paper, our goal is to develop an accurate three-dimensional (3D) finite element model for myopic cases after ISPRK, which would lead to improve the total outcome of the procedure. The model was designed with typical dimensions, material properties, boundary conditions, and loads. Model outcomes were compared with the 2D model created by Bryant et al. ${ }^{8}$ to determine its accuracy. We also provide a 3D finite element model for a virtual astigmatic case to visualize the effects on corneal curvature and shape. The materials and methods used in this work are explained in the following section while the obtained results together with their discussion are given in Sec. 3. We finally end this paper with a conclusion and future work.

\section{Materials and Methods}

Finite element modeling is a suitable method for studying many practical problems prior to realization. Since the process of ISPRK is a very recent method of sight correction, more quantification and verification of its performance are still 
needed. Therefore, finite element modeling was used to simulate the ISPRK procedure in this problem, as it has the capability for incorporating biomechanical properties. In other words, we dealt with this problem as a pure biomechanical problem.

The model was based on the following assumptions:

(1) Axisymmetric 3D model. We assumed that modeling in 3D would enhance the accuracy of the model, due to the application of the loads and boundary conditions in $3 \mathrm{D}$ vectors, which eliminates any approximations needed for the previous $2 \mathrm{D}$ models. In addition, the model in $3 \mathrm{D}$ facilitated modeling astigmatic cases, as will be shown.

(2) Isotropic incompressible material, with exponential elastic stress-strain relationship for the cornea, this assumption was based on the study by Bryant et $a .^{8}$

(3) The cavity collapse control was performed using contact and target elements (contact pair) on the cavity's posterior and anterior surfaces, respectively. The contact pair was set to close the intrastromal cavity and allow sliding between the closed surfaces.

(4) Fixed nodal displacements at the borders of the cornea (limbus), which are considered as the boundary conditions. Internal intraocular pressure of $15 \mathrm{mmHg}$, which is considered the load.

(5) No temperature effects were included in the model. The plasma induced in the stroma due to laser pulses has a temperature of about $60,000 \mathrm{~K}$, but this temperature is not to be mistaken for the local tissue temperature, since it represents the kinetic energy of the plasma electrons only in that limited volume $(10 \mu \mathrm{m})$ of stroma, which it is very unlikely to affect the surrounding tissue. $^{2}$

In the following subsections, we describe the implementation of the model, which was implemented using ANSYS software package, version 10 (ANSYS Inc., Canonsburg, South Carolina, USA).

\subsection{Model description}

A typical finite element analysis consists of three stages, namely; preprocessing, processing, and post-processing. The preprocessing stage consists of geometry building, incorporating material properties, meshing (i.e., creating elements and nodes then assembling elements to represent the entire object), and finally applying boundary conditions and loads. The processing stage consists of solving a set of equations simultaneously to obtain nodal results, which are mainly the nodal displacements. The post-processing stage incorporates graphical representation of the solution and calculating the corneal power change in Diopters. A complete finite element analysis is the sequence of these three stages. In the following subsections, we describe the application of each stage to our model. 


\subsubsection{Governing equation}

The finite element method divides the object to be modeled into shaped region, or what is called elements. Each element consists of a number of nodes, which varies according to the element type. An element can have the behavior of a structural element, fluid, heat, or other physical behavior according to the problem given. An approximate solution for the equation governing the behavior of that element can be developed for each of the elements forming the whole object. The total solution is then generated by linking together or assembling the individual solutions taking care to ensure continuity at the boundaries. The equation governing the behavior of the element must be developed; this equation represents a fit of the function to the solution of the underlying differential equation. Mathematically, the resulting element equation will often consist of a set of linear algebraic equations that can be expressed in matrix form, as follows:

$$
[K] *[U]=[F],
$$

where $[\mathrm{K}]$ is called element stiffness matrix or the property matrix, $[\mathrm{U}]$ a column vector of the nodal displacements of the element, and [F] a column vector reflecting the effect of any external influences applied at the nodes, which are the boundary conditions and loads. For the model in hand, which is purely structural, the governing equation is the stress-strain relationship for the cornea, which its basic form is:

$$
[\sigma]=[\nu] \times[\varepsilon],
$$

where $[\sigma]$ is the stress vector, and $[\nu]$ elasticity matrix, and $[\varepsilon]$ strain vector. This equation is used to find the stiffness matrix $[\mathrm{K}]$. In addition, for a material under load, the element strain energy $\Lambda$ is:

$$
\Lambda=\frac{1}{2} \int_{V}[\sigma]^{T}[\varepsilon] d V=\frac{1}{2} \int_{V}[\varepsilon]^{T}[\nu]^{T}[\varepsilon] d V .
$$

The last equation is used to obtain the element stiffness matrix $[\mathrm{K}]$ in terms of the elasticity matrix $[\nu]$. The detailed mathematical procedure is out of this paper's scope.

Then, all the individual equations in the form of Eq. (1) are assembled, to obtain equation of the entire object, which is expressed as:

$$
\left[\mathrm{K}^{\prime}\right]\left[\mathrm{U}^{\prime}\right]=\left[\mathrm{F}^{\prime}\right]
$$

where $\left[\mathrm{K}^{\prime}\right]$ is called the object property matrix, and $\left[\mathrm{U}^{\prime}\right]$ and $\left[\mathrm{F}^{\prime}\right]$ are column vectors for the unknowns and the external forces, respectively; which are marked with primes to denote that they are an assemblage of the vectors $[\mathrm{U}]$ and $[\mathrm{F}]$ from the individual elements. After that, the boundary conditions and loads are incorporated in $\left[\mathrm{F}^{\prime}\right]$. The solution stage, mathematically, is done through solving Eq. (4) for nodal displacements $\left[\mathrm{U}^{\prime}\right]$. The solution is performed by the use of well-known matrix solving methods. ${ }^{12}$ 
Table 1. Values of parameters used in the corneal model.

\begin{tabular}{lcc}
\hline Parameter & Value or equation & References \\
\hline $\begin{array}{l}\text { Index of refraction } \\
\text { Material properties }\end{array}$ & 1.376 & Ref. 11 \\
$\begin{array}{l}\text { Poisson's ratio } \\
\text { Density }\end{array}$ & 0.49 & Ref. 8 \\
Stress-strain relationship & $1.4^{*} 10^{-6} \mathrm{Kg} / \mathrm{mm}^{3}$ & Ref. 13 \\
$\quad$ Geometry & $17.5 * 10^{-4}\left(e^{48.3 * \varepsilon}-1\right)$ & Ref. 8 \\
Anterior radius of curvature & $7.6 \mathrm{~mm}$ & Ref. 8 \\
Posterior radius of curvature & $6.7 \mathrm{~mm}$ & Ref. 8 \\
Thickness at apex & $0.5 \mathrm{~mm}$ & Ref. 8 \\
Thickness at limbus & $0.65 \mathrm{~mm}$ & Ref. 8 \\
Diameter & $11.5 \mathrm{~mm}$ & Ref. 8 \\
Corneal edges inclination & $40^{\circ}$ & Ref. 14 \\
\hline
\end{tabular}

\subsubsection{Geometry and material properties}

The used material properties and geometric parameters in our finite element model are listed in Table 1. Those numbers are average values for the normal human eyes. We used the geometric parameters to build up our object's geometry in 3D; which is the cornea. The material properties were incorporated in the object property matrix $\left[K^{\prime}\right]$.

\subsubsection{Meshing}

The element type to fill out our object was selected carefully to fulfill the following conditions:

(1) Compliance with the physics of the current problem, which is structural.

(2) 3D geometry, with 3D translational degrees of freedom.

(3) Behavior of the element, e.g. deflection, plasticity, etc., as there are a preferred element types for a specific application type, our main behavior here is deflection.

(4) Sufficient number of nodes, in which we have a trade off, as the number of nodes increases accuracy is improved and at the same time computational cost, and vice versa; therefore, one has to select an "enough number" of nodes per element. Another issue is that we must select an element with an "enough number" of nodes to guarantee that the mesh created for an irregular geometry is correct and well fitted, as the increased number of nodes makes the element more flexible to fit an irregular and complex part of the object being meshed.

Imposing the above conditions, making a survey in the ANSYS elements library, and trying a number of elements before deciding which element is best suited for our problem, an element type from the SOLID family, with 10 nodes was selected.

After selecting a suitable element type, we had then to fill out the whole object with elements, or to mesh it. The main meshing parameters were element size and 
element shape, in which we selected the tetrahedral shape with sufficient element size, which fitted more the irregular geometry of the cornea, especially the cavity part irregularities. There are other meshing options that we have used through the software package, like smart sizing, in which smaller elements are created at the small edges, especially the cavity edges, and normal elements at normal edges.

\subsubsection{Cavity collapse effect}

A crucial event that must be considered is the intrastromal cavity collapse during the processing stage. The elements attached to the cavity surfaces, anterior and posterior, would be attached to each other during the solution of the model, and continue at that state till the solution is over, which is the main effect that results in corneal curvature change. Structural elements do not feel each other unless they were fixed from the meshing stage; therefore, we have to make the intrastromal cavity boundaries elements understand the proximity rules. There are two approaches of doing so, one by creating gap elements between nodes that are expected to be attached to each other, that approach were used by Bryant et al. in their model. ${ }^{8}$ Another approach is to use special types of elements that are called contact and target elements. Their behavior is to calculate the proximity between elements and to determine if contact occurred between them or not; if occurred, then, the contact and target elements should close the gap between elements or make the attached elements slide upon each other or both effects; closing the gap and sliding. We used that later approach and made what is called a "contact pair" of elements. That pair contains two types of elements, one is the contact type that is attached to the lower surface of the cavity, and the other one is the target type that is attached to the upper surface of the cavity. This orientation resembles the movement of the cavity closure. Those elements are applicable to 3D structural problems. The contact parameters are set to determine the behavior of the contact pair, which is to entirely close the intrastromal cavity and allow sliding between the contact pair upon the detection of contact, based upon the position of the contact elements nodes. This concludes the meshing process, which gave us the meshed object in Fig. 1. Note the concentrated elements at the center of the cornea that result from filling the irregular intrastromal cavity with elements in a good way, and also the attached contact elements to them.

\subsubsection{Loads and boundary conditions}

The next step is to apply loads and boundary conditions on the meshed object, to make the elements know how to behave and deform upon those conditions. The load throughout the model is the IOP of the eye ball affecting the posterior surface of the cornea. This pressure ranges from 10 to $21 \mathrm{mmHg}$ in normal eyes, with an average of $15 \mathrm{mmHg}\left(2 \times 10^{-3} \mathrm{~N} / \mathrm{mm}^{2}\right)$. Previous work on ISPRK modeling used this value for IOP, ${ }^{8}$ therefore, to compare the results, one should use the same loading value. 


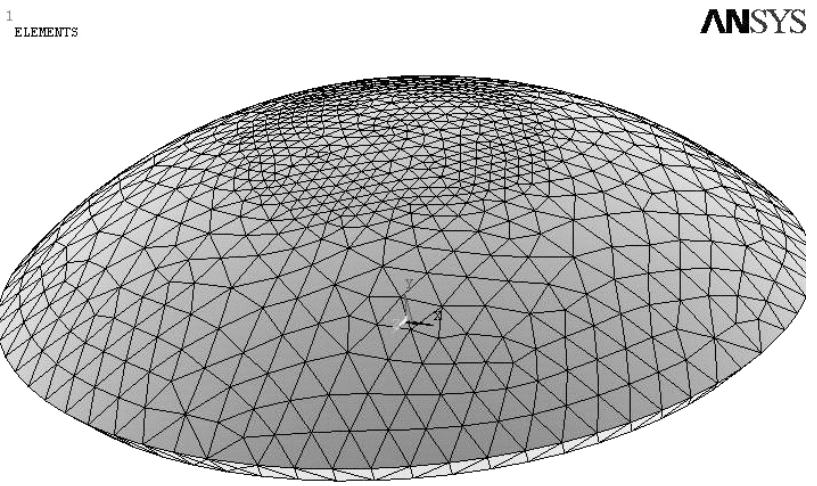

Fig. 1. Corneal mesh.

The applied boundary conditions here include two parts. The first was discussed in the previous subsection, which is the contact between the elements resembling the intrastromal cavity surfaces. The other is the connection between sclera and cornea, which is represented by the $40^{\circ}$ inclined part of the geometry, and fixing that part in all directions, ${ }^{10}$ i.e. fixing the limbus of the cornea. That is accomplished by limiting the degrees of freedom for the nodes attached to the limbus. That limitation is translated to zero translational $x, y$, and $z$ degrees of freedom.

The load and boundary conditions were applied in the myopic and astigmatic models as well, with the same values.

\subsubsection{The model in steps}

First, the modeling process was started by determining the physics of the problem, which was a structural problem, and then the corneal geometry and material properties was entered to the model. After that, a suitable element type was selected that was used in the meshing step. The quality of the elements in terms of size and fitness to the cornea was tested and checked. Then, a contact pair was created between the intrastromal cavity surface elements, so as to ensure that the elements did not overlap upon collapse of the cavity. Following that, the IOP load and the boundary conditions were applied to the corneal model. Thus, the solution phase was begun in which nodal displacements were calculated. Then, the graphical results were displayed and checked regarding nodal displacement profile to be logically acceptable and contact pair status to be fully closed. The corrected corneal refractive power was then calculated; as will be shown in the next section, and compared with a reference corneal power, which is the corneal power before laser treatment, i.e. preoperative power. After the final results were obtained, they were checked and if they were unacceptable, we had to get back through the process starting from the "geometry" step, and review everything and make necessary adjustments, especially with regard to the contact elements attached to the cavity 


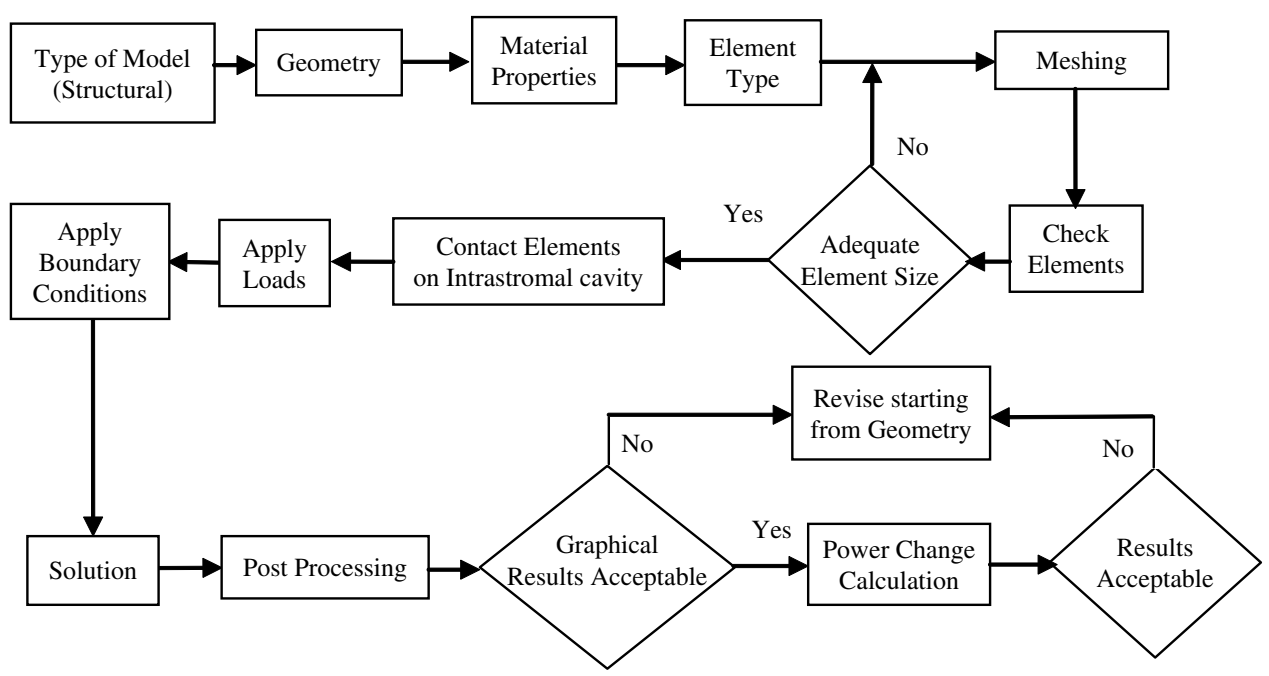

Fig. 2. Block diagram of corneal modeling.

elements, in terms of the full closure of the cavity. The finite element model block diagram is shown in Fig. 2.

\subsection{Modeling myopic cases}

The previously described finite element model was used to simulate and predict the outcome of treating myopic cases with ISPRK. First, the model was run without including the cavity. In other words, the model was run preoperatively to calculate preoperative corneal power, $\mathrm{D}$ in diopters. Then, the model was run after including the cavity collapse effect, i.e. postoperatively, and the postoperative corneal power, Dp, was calculated and compared with the preoperative value. The postoperative power correction was the difference between $\mathrm{D}$ and $\mathrm{Dp}$.

Figure 3(a) shows the corneal model after completing the preprocessing phase of the model. Figure $3(\mathrm{~b})$ shows the postoperative displacement profile of the anterior surface of the cornea. This profile shows minimal displacement at the limbus and maximum at the part facing the cavity border inside the cornea (the bright ring of the profile), and then moderate displacement is observed at the center part. This profile is the main idea behind any photorefractive eye surgery, as it causes the corneal radius of curvature to be bigger, which lowers the corneal power to correct myopia.

In order to calculate the postoperative corneal radius of curvature, the finite element model output was used. The nodal postoperative displacement, which is the primary output of the model, was added to the original nodal coordinates to get the postoperative nodal coordinates $\left(X_{p}, Y_{p}\right)$ of the central portion of the cornea, 


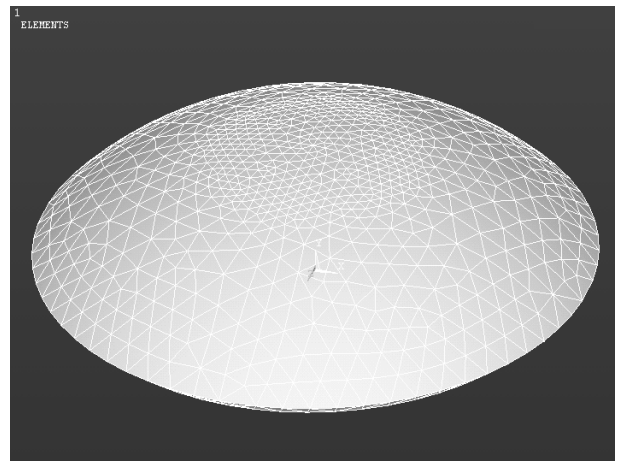

(a)

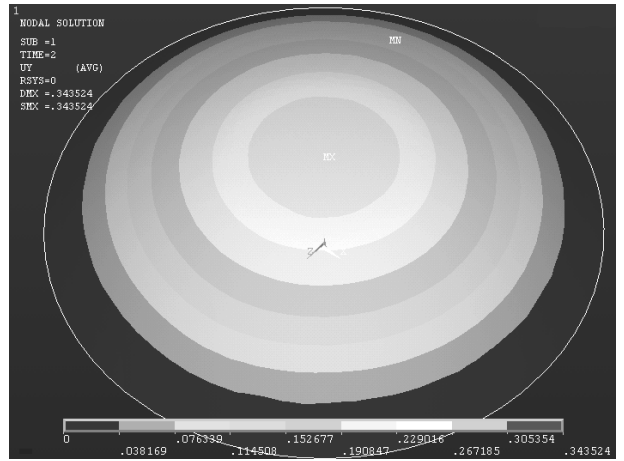

(b)

Fig. 3. Myopic model. (a) Finite element model of the human cornea and (b) Postoperative displacement profile of the anterior surface of the cornea.

which is the central $3 \mathrm{~mm}$ of the anterior corneal surface. This zone was considered to be the optical zone that the light passes through to reach the retina.

Then, the curve fitting method was employed to fit a circle with radius $\mathrm{R}$ on the central nodes. The used curve fitting function is:

$$
F=\sum_{i=1}^{n}\left[R^{2}-\left[\left(\mathrm{Xp}_{i}-\mathrm{a}\right)^{2}+\left(\mathrm{Yp}_{i}-\mathrm{b}\right)^{2}\right],\right.
$$

where $\mathrm{a}$ and $\mathrm{b}$ are constants representing the center of the fitted circle. Substituting $\mathrm{R}$ into the next relationship gives the postoperative corneal power, $\mathrm{Dp}$ in diopters, which is the commonly used unit to measure the corneal optical refractive power:

$$
D p=\frac{(n-1)}{R}=\frac{0.376}{R} .
$$

Here, $n$ is the corneal index of refraction. In addition, the corneal power change, $\Delta D$ is:

$$
\Delta D=D p-D
$$

As mentioned earlier, D is the reference value of the corneal power resulting from running the previous model without taking the intrastromal cavity into consideration.

Using this methodology, the model was verified by comparing its output with three clinical categorized cases, and also with an earlier model, ${ }^{8}$ which is shown in the results section.

\subsection{Modeling astigmatic cases}

As an extension to the myopic 3D finite element model, we applied it to simulate and predict an astigmatic case combined with myopia. A virtual (supposed) case was created, in which 7.4 diopters of power difference between two perpendicular corneal 
meridians in the $X$ and $Z$ directions, i.e. cylindrically shaped corneal surface. The cornea also has a myopic effect of seven diopters that needs to be corrected. An intrastromal cavity was proposed to correct that specific case with dimensions based on the general rule of thumb used by some eye laser surgeons, that every one diopter of myopia is treated with about $13 \mu \mathrm{m}$ of ablation depth. ${ }^{15}$ In addition, the cavity was oriented so that the weaker power meridian of the cornea was opposing the more power correcting meridian of the cavity.

The astigmatic model differs from the pure myopic model in the geometry building step, and the post-processing steps were modified accordingly. Other steps were nearly the same. Figure 4(a) shows the proposed intrastromal cavity for treating an astigmatic myopic case and Fig. 4(b) shows the displacement profile of the anterior surface of the cornea after running the model. This profile presents the idea behind treating astigmatism using laser procedures, in which two corneal meridians are deformed with different degrees, so that corneal power is changed differently at those meridians and the corneal surface becomes more spherical rather than cylindrical.

Calculating the postoperative power change was based on the same method used in calculating power for myopia, except that the corneal radius of the curvature was obtained in the $X$ and $Z$ directions, represented by $R x$ and $R z$. The nodes in the central $3-\mathrm{mm}$ zone were also considered in the calculations. The curve fitting equations in this case are:

$$
\begin{aligned}
& F_{1}=\sum_{i=1}^{n}\left[R x^{2}-\left[\left(\mathrm{Xp}_{i}-\mathrm{a}_{1}\right)^{2}+\left(\mathrm{Yp}_{i}-\mathrm{b}_{1}\right)^{2}\right]\right. \\
& F_{2}=\sum_{i=1}^{n}\left[R z^{2}-\left[\left(\mathrm{Zp}_{i}-\mathrm{a}_{2}\right)^{2}+\left(\mathrm{Yp}_{i}-\mathrm{b}_{2}\right)^{2}\right] .\right.
\end{aligned}
$$

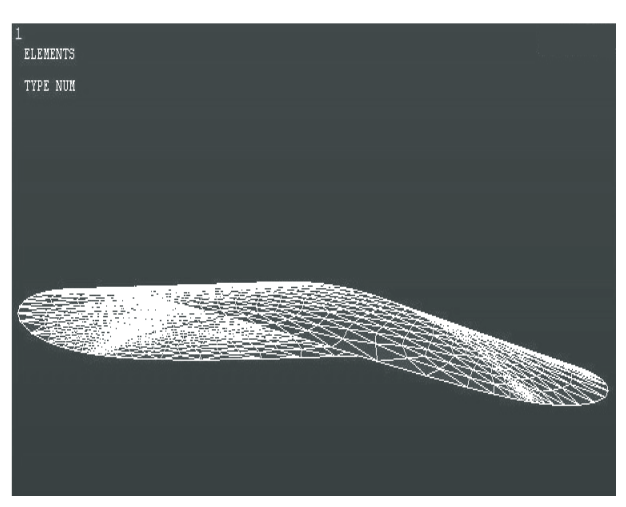

(a)

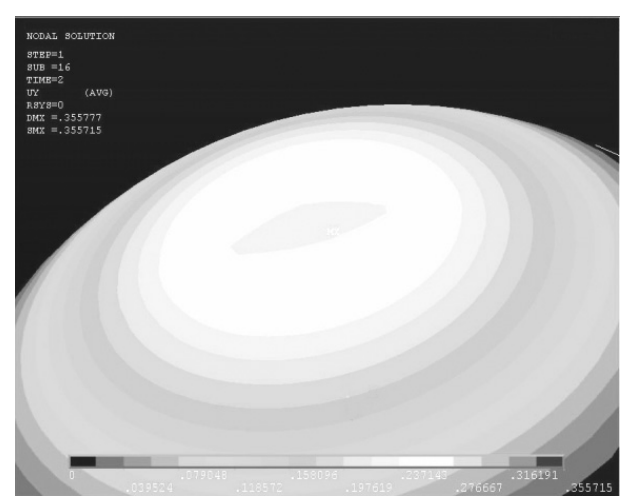

(b)

Fig. 4. As tigmatic model: (a) meshed intrastromal cavity proposed to treat astigmatic case and (b) displacement profile of the anterior surface of the cornea having astigmatism treated. 
Here $\mathrm{a}_{1}$ and $\mathrm{a}_{2}$ and $\mathrm{b}_{1}$ and $\mathrm{b}_{2}$ are constants representing the centers of the fitted circles. Substituting $R x$ and $R z$ in the next equations gives us the postoperative corneal refractive powers, $D x p$ and $D z p$ respectively.

$$
\begin{gathered}
D x p=\frac{(n-1)}{R x}=\frac{0.376}{R x}, \\
D z p=\frac{(n-1)}{R z}=\frac{0.376}{R z} .
\end{gathered}
$$

Here, $n$ is the corneal index of refraction. The corneal power changes in the $X$ and $Z$ directions are:

$$
\begin{aligned}
& \Delta D x=D x p-D x \\
& \Delta D z=D z p-D z .
\end{aligned}
$$

Where $D x$ and $D z$ are the preoperative corneal powers in diopters. Equation (10.a) represents the myopic power correction.

The resultant astigmatic power correction is:

$$
\Delta \text { Dast }=D x p-D z p .
$$

\section{Results and Discussion}

\subsection{Myopic model}

Running the myopic model with three different intrastromal cavity dimensions (different diameters and thicknesses) results in Table 2. Those dimensions and corneal power changes are categorized averages of 10 clinical cases, in which the first row in the table is the average of two clinical cases with the same cavity dimensions, the second is the average of six cases and the third is the average of two cases. They were used to compare the outcome of the model with a previous work, namely the 2D model created by Bryant et al., where these clinical data were taken from their published work. ${ }^{8}$

Comparing the results with the $2 \mathrm{D}$ finite element model created by Bryant et al. shows that our model is more correlated to the clinical data, with a smaller percentage of error, about $2.5 \%$ compared to $23.5 \%$. The main reasons behind that improvement in results are the followings:

(1) Using contact and target elements to control the cavity collapse event during the run of the model, instead of using gap elements between the surfaces of the

Table 2. Model trials and comparisons with clinical data and a 2D model.

\begin{tabular}{ccccccr}
\hline $\begin{array}{c}\text { Cavity } \\
\text { diameter }(\mathrm{mm})\end{array}$ & $\begin{array}{c}\text { Cavity } \\
\text { thickness }(\mu \mathrm{m})\end{array}$ & $\begin{array}{c}\text { Power change } \\
\text { (diopters) }\end{array}$ & $\begin{array}{c}\text { 2D model } \\
\text { (diopters) }\end{array}$ & Error & $\begin{array}{c}\text { 3D model } \\
\text { (diopters) }\end{array}$ & Error \\
\hline 4 & 100 & 11.300 & 14.500 & $\mathbf{3 . 2 0 0}$ & 11.700 & $\mathbf{0 . 4 0 0}$ \\
4 & 120 & 13.083 & 16.700 & $\mathbf{3 . 6 1 7}$ & 13.000 & $\mathbf{- 0 . 0 8 3}$ \\
3.2 & 120 & 19.6 & 22.5 & $\mathbf{2 . 9 0 0}$ & 19.000 & $\mathbf{- 0 . 6 0 0}$ \\
\hline
\end{tabular}


cavity. The main advantages are having a surface-to-surface contact pair, not a node-to-node one, and the absence of any need to determine a gap element stiffness value that could result in improper behavior.

(2) Creating a 3D model, so no approximations were made regarding the affecting pressure. That means that the pressure on the posterior surface of the cornea was applied with the same clinical value of $15 \mathrm{mmHg}$ in the model. This had not been the case with the 2D model, which had used shell theory to model pressurized objects, and in that case pressure was applied on a line not an area.

(3) The approach used to create the cavity shape inside the stroma. Both approaches were mentioned in the ISPRK United States Patent. ${ }^{6}$ The geometric model guided one was used by the $2 \mathrm{D}$ model and the corneal curvatures averaging guided one was used in this $3 \mathrm{D}$ model.

(4) Using a different value for the index of refraction of the cornea that can be found at the references, and incorporating corneal density into the model as an additional material property.

As an additional verification of the current model, another three experiments were conducted with the same cavity dimensions and overall parameters, but with increasing the IOP from $15 \mathrm{mmHg}\left(2 \times 10^{-3} \mathrm{~N} / \mathrm{mm}^{2}\right)$ to $20 \mathrm{mmHg}(2.667 \times$ $10^{-3} \mathrm{~N} / \mathrm{mm}^{2}$ ). Table 3 illustrates the second three trials compared with the first ones.

From the previous predictions, we found that increasing the IOP results in a decrease in the corneal refractive power with about 0.6 diopters. Generally speaking, those values agree with previous studies on the IOP increase effect. ${ }^{11,16}$

\subsection{Astigmatic model}

Table 4 summarizes the results of the astigmatic myopic case model, and the calculations made using Eq. (8)-(11):

Table 3. Effect of changing the IOP on the model outcome.

\begin{tabular}{cccc}
\hline Cavity dimensions & Predicted power $15 \mathrm{mmHg}$ & Predicted power $20 \mathrm{mmHg}$ & Difference \\
\hline 4,100 & 11.7 & 11.1 & -0.6 \\
4,120 & 13 & 12.5 & -0.5 \\
$3.2,120$ & 19 & 18.35 & -0.65 \\
\hline
\end{tabular}

Table 4. Astigmatic model outcomes and the resultant corrections.

\begin{tabular}{lccc}
\hline & $\begin{array}{c}\text { Preoperative } \\
\text { values (D) }\end{array}$ & $\begin{array}{c}\text { Postoperative } \\
\text { values (D) }\end{array}$ & $\begin{array}{c}\text { Directional power } \\
\text { correction }\end{array}$ \\
\hline Power $X$ direction & 48.2 & 41.3 & -6.9 \\
Power $Z$ direction & 55.64 & 39.17 & -16.47 \\
Astigmatic power & & & \\
correction ( $\Delta$ Dast) & -7.44 & 2.13 & \\
\hline
\end{tabular}


We noted that the myopic correction was nearly achieved, with only an error of 0.1 diopters from the target value in the $X$ direction (seven diopters). In addition, for the astigmatism correction, there is a bigger shift of 2.13 diopters from the target value of zero diopters, which is the pure spherical case for the cornea. This indicates that an overcorrection occurred for the astigmatism effect correction, which needs to be modified regarding the intrastromal cavity thickness in the $Z$ direction by making it thinner in that direction. This demonstration of the model for that type of clinical cases shows the usefulness and importance of the model as a preoperative predictive tool and as a planning guide.

\section{Conclusions}

An accurate finite element model used for simulating myopic ISPRK laser procedures is developed and verified. Based on the comparison of the model predictions with actual clinical data, it was shown that this type of procedures can be predicted, taking into consideration the related factors affecting corneal power changes, such as the IOP, specific material properties for individual cases, and other factors. This work showed that astigmatism can also be modeled and predicted by using nearly the same myopic model outlines. A virtual astigmatic case was introduced to illustrate the model, and used to evaluate the suitability of a proposed intrastromal cavity shape. This model can be used as a preoperative planning tool for the eye surgeon that its usage would improve the outcome of the surgery by selecting the most appropriate parameters for a specific case. Future work will be conducted to implement a more accurate model including incorporation of corneal anisotropy, use of probabilistic models, and inclusion of the wound healing effect by the use of time-dependent simulation to the healing process.

\section{References}

1. http://www.fpnotebook.com/EYE.htm, Family practice notebook Web site.

2. Wolbarsht ML, Laser Applications in Medicine 8 Biology, 4th edn., Plenum Pub Corp, New York, 2000.

3. http://www.eyemdlink.com/EyeProcedure.asp?EyeProcedureID=5, Web site.

4. Hasson M, Femtosecond intrastromal correction of myopia a viable option, Ocular Surg News 27(14):6-6, 2009.

5. Ratkay-Traub I, Ferincz IE, Juhasz T, Kurtz RM, Krueger RR, First clinical results with the femtosecond neodynium-glass laser in refractive surgery, $J$ Refract Surg 19(2):94-103, 2003.

6. Juhasz T, Bille JF, Intrastromal Photorefractive Keratectomy, United States Patent No. 5993438, 1999.

7. Vogel A, Capon MR, Asiyo-Vogel MN, Birngruber R, Intraocular photodisruption with picosecond and nanosecond laser pulses: Tissue effects in cornea, lens, and retina, Invest Ophthalmol Vis Sci 35(7):3032-3044, 1994.

8. Bryant MR, Marchi V, Juhasz T, Mathematical models of picosecond laser keratomileusis for high myopia, J Refract Surg 16(2):155-162, 2000. 
9. Yee K, Intrastromal Refractive Correction Systems and Methods, United States Patent No. 0234335, 2009.

10. Hennighausen H, Bille JF, Computer simulation of corneal curvature change caused by intrastromal ablations using a picosecond laser system, Proc SPIE 2330:5-13, 1994.

11. Deenadayalu C, Mobasher B, Rajan SD, Hall GW, Refractive change induced by the LASIK flap in a biomechanical finite element model, J Refract Surg 22(1):286-292, 2006.

12. Hutton DV, Fundamentals of Finite Element Analysis, McGraw-Hill, 2004.

13. Power ED, Stitzel JD, West RL, Herring IP, Duma SM, A nonlinear finite element model of the human eye for large deformation loading, Proceedings of 25th Annual Meeting of the American Society of Biomechanics, San Diego, 2001.

14. Anderson K, El-Sheikh A, Newson T, Application of structural analysis to the mechanical behaviour of the cornea, J R Soc Interface 1(1):3-15, 2004.

15. Huang D, Tang M, Shekhar R, Mathematical model of corneal surface smoothing after laser refractive surgery, Am J Ophthalmol 135(1):267-278, 2003.

16. Crouch JR, Merriam JC, Crouch ER, Finite element model of cornea deformation, MICCAI 8(2):591-8, 2005.

17. Bathe KJ, Finite Element Procedures, Prentice-Hall, New Jersey, 1996. 